1912.] EXISTENCE THEOREM FOR IMPLICIT FUNCTIONS. 175

\title{
A NEW PROOF OF THE EXISTENCE THEOREM FOR IMPLICIT FUNCTIONS.
}

\author{
BY PROFESSOR GILBERT AMES BLISS.
}

(Read before the American Mathematical Society October 28, 1911.)

The theorem with which this paper has to do is the one which states the existence of a set of functions

$$
y_{i}=y_{i}\left(x_{1}, x_{2}, \cdots, x_{m}\right) \quad(i=1,2, \cdots, n)
$$

which satisfy a system of equations of the form

$$
f_{i}\left(x_{1}, x_{2}, \cdots, x_{m} ; y_{1}, y_{2}, \cdots, y_{n}\right)=0 \quad(i=1,2, \cdots, n) .
$$

For the case in which the functions $f$ are only assumed to be continuous and to have continuous first derivatives, the proof seems to have been originally given by Dini.* His method is to show the existence of a solution of a single equation, and then to extend his result by mathematical induction to a system of the form given above, a plan which has been followed, with only slight alterations and improvements in form, by most writers on the theory of functions of a real variable. In a more recent paper $\dagger$ Goursat has applied a method of successive approximation which enabled him to do away with the assumption of the existence of the derivatives of the functions $f$ with respect to the independent variables $x$.

One can hardly be dissatisfied with either of these methods of attack. It is true that when the theorem is stated as precisely as in the following paragraphs, the determination of the neighborhoods at the stage when the induction must be made is rather inelegant, but the difficulties encountered are not serious. The introduction of successive approximations is an interesting step, though it does not simplify the situation and indeed does not add generality with regard to the assumptions on the functions $f$. The method of Dini can in fact, by only a slight modification, be made to apply to cases where the functions do not have derivatives with respect to the variables $x$.

* Lezioni di Analisi infinitesimale, vol. 1, chap. 13. For historical remarks, see Osgood, Encyclopädie der mathematischen Wissenschaften, II, B 1, \& 44 and footnote 30 .

$\dagger$ †ulletin de la Société mathématique, vol. 31 (1903), page 185. 
176 EXISTENCE THEOREM FOR IMPLICIT FUNCTIONS. [Jan.,

The proof which is given in the following paragraphs seems to have advantages in the matter of simplicity over either of the others. It applies equally well, without induction, to one or a system of equations, and requires only the initial assumptions which Goursat mentions in his paper.

Where it is possible without sacrificing clearness, the row letters $f, x, y, p, a, b$ will be used to denote the systems

$$
\begin{aligned}
f & =\left(f_{1}, f_{2}, \cdots, f_{n}\right), & x & =\left(x_{1}, x_{2}, \cdots, x_{m}\right), \\
y & =\left(y_{1}, y_{2}, \cdots, y_{n}\right), & a & =\left(a_{1}, a_{2}, \cdots, a_{m}\right), \\
b & =\left(b_{1}, b_{2}, \cdots, b_{n}\right), & p & =\left(a_{1}, a_{2}, \cdots, a_{m} ; b_{1}, b_{2}, \cdots, b_{n}\right) .
\end{aligned}
$$

In this notation the equations (1) have the form

$$
f(x ; y)=0,
$$

the interpretation being that every element of $f$ is a function of $x_{1}, x_{2}, \cdots, x_{m} ; y_{1}, y_{2}, \cdots, y_{n}$, and every $f_{i}$ is to be set equal to zero. The notations $p_{e}, a_{\epsilon}, b_{e}$ represent respectively the neighborhoods

$$
|x-a|<\epsilon,|y-b|<\epsilon ; \quad|x-a|<\epsilon ; \quad|y-b|<\epsilon
$$

of the points $p, a, b$.

With these notations in mind the fundamental theorem which is to be proved may be stated as follows:

Hypotheses:

1) the functions $f(x ; y)$ are continuous, and have first partial derivatives with respect to the variables $y$ which are also continuous, in a neighborhood of the point $p$;

2) $f(a ; b)=0$

3) the functional determinant $D=\partial\left(f_{1}, f_{2}, \cdots, f_{n}\right) / \partial\left(y_{1}, y_{2}\right.$, $\left.\cdots, y_{n}\right)$ is different from zero at $p$.

Conclusions:

1) a neighborhood $p_{\boldsymbol{e}}$ can be found in which there corresponds to a given value $x$ at most one solution $(x ; y)$ of the equations $f(x ; y)=0$

2) for any neighborhood $p_{\mathrm{e}}$ with the property just described a constant $\delta \leqq \epsilon$ can be found such that every $x$ in $a_{\delta}$ has associated with it a point $(x ; y)$ which satisfies the equations $f(x ; y)=0$;

3 ) the functions $y\left(x_{1}, x_{2}, \cdots, x_{m}\right)$ so found are continuous in the region $a_{\delta}$. 
For the neighborhood $p_{\mathrm{e}}$ let one be chosen in which the continuity properties of the functions $f$ are preserved. If $(x ; y)$ and $\left(x ; y^{\prime}\right)$ are two points in $p_{e}$, it follows by applying Taylor's formula to the differences $f\left(x ; y^{\prime}\right)-f(x ; y)$ that

$$
\begin{aligned}
& f_{1}\left(x ; y^{\prime}\right)-f_{1}(x ; y)=\frac{\partial f_{1}}{\partial y_{1}}\left(y_{1}{ }^{\prime}-y_{1}\right)+\cdots+\frac{\partial f_{1}}{\partial y_{n}}\left(y_{n}{ }^{\prime}-y_{n}\right), \\
& f_{n}\left(x ; y^{\prime}\right)-f_{n}(x ; y)=\frac{\partial f_{n}}{\partial y_{1}}\left(y_{1}{ }^{\prime}-y_{1}\right)+\cdots+\frac{\partial f_{n}}{\partial y_{n}}\left(y_{n}{ }^{\prime}-y_{n}\right),
\end{aligned}
$$

where the arguments of the derivatives $\partial f_{i} / \partial y_{k}$ have the form $x ; y+\theta_{i}\left(y^{\prime}-y\right)$ and $0<\theta_{i}<1$. The determinant of these derivatives is different from zero when $\left(x ; y^{\prime}\right)=(x ; y)=$ $(a ; b)$, and hence must remain different from zero if $p_{e}$ is restricted so that in it the functional determinant $D$ remains different from zero. It is then impossible that $(x ; y)$ and $\left(x ; y^{\prime}\right)$ should both be solutions of the equations $f(x ; y)=0$ if $y$ is distinct from $y^{\prime}$.

In the corresponding region $b_{\mathrm{e}}$ the function

$$
\varphi(a ; y)=f_{1}{ }^{2}(a ; y)+f_{2}{ }^{2}(a ; y)+\cdots+f_{n}{ }^{2}(a ; y)
$$

has a minimum for $y=b$, since for that value it vanishes and for every other it is positive. In particular

$$
\varphi(a ; \eta)-\varphi(a ; b)>m>0
$$

for the closed set of points $\eta$ forming the boundary of $b_{e}$, on account of the continuity of $\varphi$, and the inequality

$$
\varphi(x ; \eta)-\varphi(x ; b)>m
$$

remains true for all values $x$ in a suitably chosen domain $a_{\delta}$. Hence for a fixed $x$ in $a_{\delta}$ the minimum of $\varphi(x ; y)$ is attained at a point $y$ interior to $b_{\epsilon}$. At such a point, however,

$$
\begin{aligned}
& \frac{1}{2} \frac{\partial \varphi}{\partial y_{1}}=f_{1} \frac{\partial f_{1}}{\partial y_{1}}+f_{2} \frac{\partial f_{2}}{\partial y_{1}}+\cdots+f_{n} \frac{\partial f_{n}}{\partial y_{1}}=0, \\
& \frac{1}{2} \frac{\partial \varphi}{\partial y_{n}}=f_{1} \frac{\partial f_{1}}{\partial y_{n}}+f_{2} \frac{\partial f_{2}}{\partial y_{n}}+\cdots+f_{n} \frac{\partial f_{n}}{\partial y_{n}}=0,
\end{aligned}
$$

and this can happen only when all the elements of $f$ are zero, 
178 EXISTENCE THEOREM FOR IMPLICIT FUNCTIONS. [Jan.,

since the functional determinant $D$ is different from zero in $p_{e}$. It follows that to every point $x$ in $a_{\delta}$ there corresponds in $p_{\mathrm{e}}$ a solution $(x ; y)$ of the equations $f(x ; y)=0$.

The functions $y\left(x_{1}, x_{2}, \cdots, x_{m}\right)$ defined in this way over the region $a_{\delta}$ are all continuous. For consider the values $y$ and $y+\Delta y$ corresponding to two points $x$ and $x+\Delta x$. By applying Taylor's formula it follows from the relations

$f(x ; y+\Delta y)-f(x ; y)=f(x ; y+\Delta y)-f(x+\Delta x ; y+\Delta y)$,

which are true because $(x ; y)$ and $(x+\Delta x ; y+\Delta y)$ both make $f=0$, that

$$
\begin{aligned}
\frac{\partial f_{1}}{\partial y_{1}} \Delta y_{1}+\frac{\partial f_{1}}{\partial y_{2}} \Delta y_{2} & +\cdots+\frac{\partial f_{1}}{\partial y_{n}} \Delta y_{n} \\
& =f_{1}(x ; y+\Delta y)-f_{1}(x+\Delta x ; y+\Delta y),
\end{aligned}
$$

$$
\begin{aligned}
\frac{\partial f_{n}}{\partial y_{1}} \Delta y_{1}+\frac{\partial f_{n}}{\partial y_{2}} \Delta y_{2}+ & \cdots+\frac{\partial f_{n}}{\partial y_{n}} \Delta y_{n} \\
& =f_{n}(x ; y+\Delta y)-f_{n}(x+\Delta x ; y+\Delta y),
\end{aligned}
$$

where the arguments of the derivatives $\partial f_{i} / \partial y_{k}$ have the form $x ; y+\theta_{i} \Delta y \quad\left(0<\theta_{i}<1\right)$. The determinant of these derivatives is different from zero on account of the way in which $p_{e}$ was chosen, and the second members of the equations approach zero with $\Delta x$. Hence the same must be true of the quantities $\Delta y$, and the functions $y\left(x_{1}, x_{2}, \cdots, x_{m}\right)$ are seen to be continuous.

A similar application of Taylor's formula leads to the conclusion that

If the functions $f$ have derivatives of the first order with respect to $x_{k}$ which are continuous in the neighborhood of $p$, so have also the functions $y\left(x_{1}, x_{2}, \cdots, x_{m}\right)$ in the region $a_{\delta}$; and if the f's have all derivatives of the nth order continuous, so have the functions $y\left(x_{1}, x_{2}, \cdots, x_{m}\right)$.

For suppose

$$
\Delta x_{1} \neq 0, \quad \Delta x_{2}=\Delta x_{3}=\cdots=\Delta x_{m}=0 .
$$

Then by applying Taylor's formula to the second members of 
equations (2) it follows that

$$
\begin{aligned}
& \frac{\partial f_{1}}{\partial y_{1}} \frac{\Delta y_{1}}{\Delta x_{1}}+\frac{\partial f_{1}}{\partial y_{2}} \frac{\Delta y_{2}}{\Delta x_{1}}+\cdots+\frac{\partial f_{1}}{\partial y_{n}} \frac{\Delta y_{n}}{\Delta x_{1}}+\frac{\partial f_{1}}{\partial x_{1}}=0, \\
& \cdot \cdot \cdot \cdot \cdot \cdot \cdot \cdot \cdot \cdot \cdot \cdot \cdot \cdot \cdot \cdot \cdot \cdot \cdot \frac{\partial f_{n}}{\partial y_{n}} \frac{\Delta y_{n}}{\Delta x_{1}}+\frac{\partial f_{n}}{\partial x_{1}}=0,
\end{aligned}
$$

where the arguments of the derivatives $\partial f_{i} / \partial x_{1}$ have the form $x+\theta_{i}{ }^{\prime} \Delta x ; y+\Delta y$. Hence as $\Delta x_{1}$ approaches zero the quotients $\Delta y_{i} / \Delta x_{1}$ approach limits $\partial y_{i} / \partial x_{1}$ which satisfy the equations

$$
\begin{aligned}
& \frac{\partial f_{1}}{\partial y_{1}} \frac{\partial y_{1}}{\partial x_{1}}+\frac{\partial f_{1}}{\partial y_{2}} \frac{\partial y_{2}}{\partial x_{1}}+\cdots+\frac{\partial f_{1}}{\partial y_{n}} \frac{\partial y_{n}}{\partial x_{1}}+\frac{\partial f_{1}}{\partial x_{1}}=0, \\
& \cdot \cdot \cdot \cdot \cdot \cdot \cdot \cdot \cdot \cdot \cdot \cdot \cdot \cdot \cdot \cdot \cdot \frac{\partial f_{n}}{\partial y_{n}} \frac{\partial y_{n}}{\partial x_{1}}+\frac{\partial f_{n}}{\partial x_{1}}=0
\end{aligned}
$$

where the arguments of the derivatives of $f$ are now $(x ; y)$. A similar consideration shows the existence of the first derivatives with respect to the variables $x_{2}, x_{3}, \cdots, x_{m}$. The existence of the higher derivatives follows from the observation that the solutions of equations (3) are differentiable $n-1$ times with respect to the variables $x$ on account of the assumption that the functions $f$ are differentiable $n$ times.

\section{ON A SET OF KERNELS WHOSE DETERMINANTS FORM A STURMIAN SEQUENCE.}

BY MR. H. BATEMAN, M.A.

WEYL $^{*}$ has recently given a theorem which states that if a kernel

$$
k_{n}(s, t)=\sum_{p, i q=1}^{n} k_{p q} \Phi_{p}(s) \Phi_{q}(t) \quad\left(k_{p q}=k_{q p}\right)
$$

is formed from $n$ functions $\Phi_{p}(s)$ whose squares are integrable in the interval $(0,1)$, then the smallest positive root of the

* Göttinger Nachrichten, 1911, Heft 2, p. 110. 